\title{
Fractional Dynamics in Calcium Oscillation Model
}

\author{
Yoothana Suansook and Kitti Paithoonwattanakij \\ Faculty of Engineering, King Mongkut's Institute of Technology Ladkrabang, Chalongkrung Road, \\ Ladkrabang, Bangkok 10520, Thailand \\ Correspondence should be addressed to Yoothana Suansook; yoothana@gmail.com
}

Received 20 April 2014; Revised 8 June 2014; Accepted 1 July 2014

Academic Editor: Shaofan Li

Copyright ( 2015 Y. Suansook and K. Paithoonwattanakij. This is an open access article distributed under the Creative Commons Attribution License, which permits unrestricted use, distribution, and reproduction in any medium, provided the original work is properly cited.

\begin{abstract}
The calcium oscillations have many important roles to perform many specific functions ranging from fertilization to cell death. The oscillation mechanisms have been observed in many cell types including cardiac cells, oocytes, and hepatocytes. There are many mathematical models proposed to describe the oscillatory changes of cytosolic calcium concentration in cytosol. Many experiments were observed in various kinds of living cells. Most of the experimental data show simple periodic oscillations. In certain type of cell, there exists the complex periodic bursting behavior. In this paper, we have studied further the fractional chaotic behavior in calcium oscillations model based on experimental study of hepatocytes proposed by Kummer et al. Our aim is to explore fractional-order chaotic pattern in this oscillation model. Numerical calculation of bifurcation parameters is carried out using modified trapezoidal rule for fractional integral. Fractional-order phase space and time series at fractional order are present. Numerical results are characterizing the dynamical behavior at different fractional order. Chaotic behavior of the model can be analyzed from the bifurcation pattern.
\end{abstract}

\section{Introduction}

The behaviors of many physical systems are nonlinear. The study of complexity arising from nonlinear systems is an intrigue subject for scientific research. Nonlinear systems have demonstrated a wide range of strange behaviors; the small change in initial conditions or system parameters result in long-term unpredictable behavior known as chaos.

Nonlinear dynamical systems have many interesting behaviors to study. The research in this field starts from the discovery of chaos in atmospheric convection model by Lorenz [1]. The equations that described the dynamical system are differential equations which yield different type of solutions [2] such as limit cycle, periodic, periodic doubling, nonperiodic, and chaotic solutions. Further studies in this field are chaos synchronization [3] and fractional-order dynamical system [4-17].

The biological systems are examples of nonlinear system where chaos arises including cardiac rhythms and bursting patterns of excited neural and intracellular chemical oscillation [18]. Nonlinear dynamical systems in living organisms are examples of complex system which evolved far from equilibrium conditions [19]. Many biological phenomena can now be understood with mathematical modeling [18]. The studies biological systems via nonlinear dynamics are feasible to discover new explanation in biological sciences and emerging applications for clinical diagnosis and therapy [19]. The examples of nonlinear dynamics in living organism include respiratory burst, glycolysis, peroxidase reaction, and $\mathrm{Ca}^{2+}$ oscillations $[18,19]$.

Biorhythms are example of nonlinear dynamics in living organism, usually associated with excitable cells, but there is growing evidence that nonexcitable cells may also exhibit periodic behavior [20]. Much of this periodic activity is driven by regular oscillations in intracellular calcium $[19,20]$. The experimental studies show that $\mathrm{Ca}^{2+}$ oscillations are the changing in free cytosolic $\mathrm{Ca}^{2+}$ concentration, which are found in various cell types [21].

A nonlinear dynamical system is a deterministic system that is feasible to explain by set of differential equations [2]. Recently, studies on theory of fractional calculus have been applied to describe the dynamical system where the derivative can be fractional order [4-17]. 


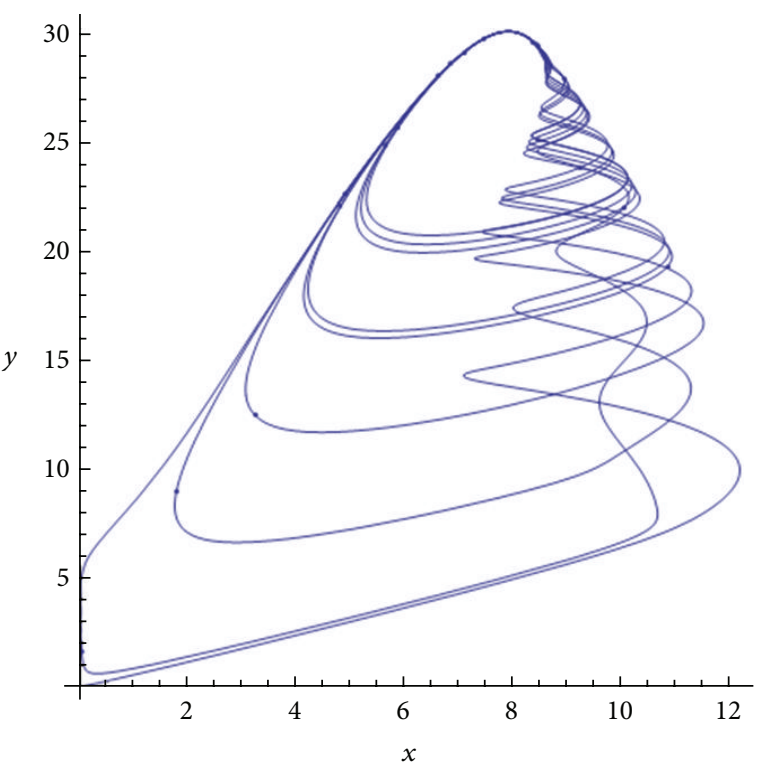

(a)

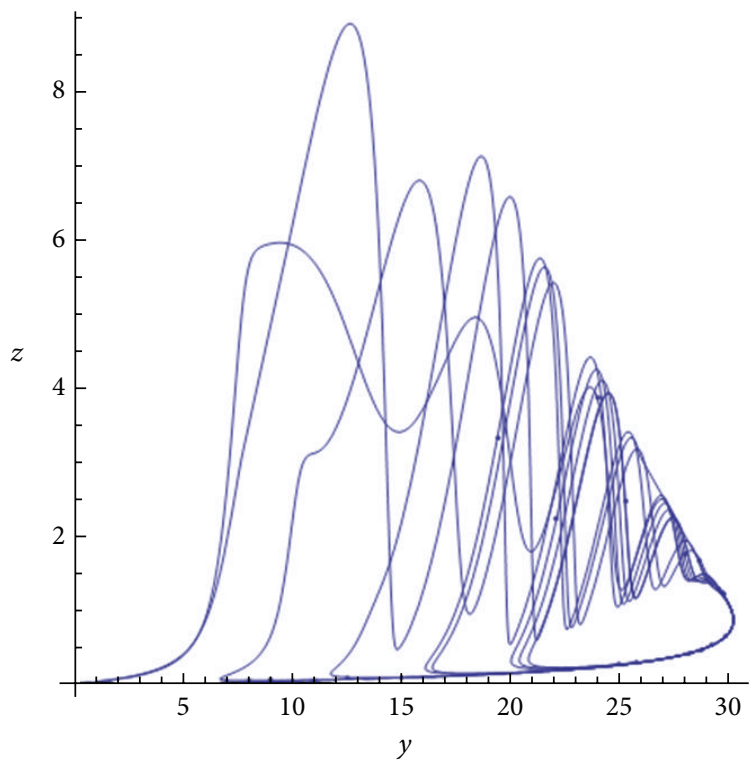

(c)

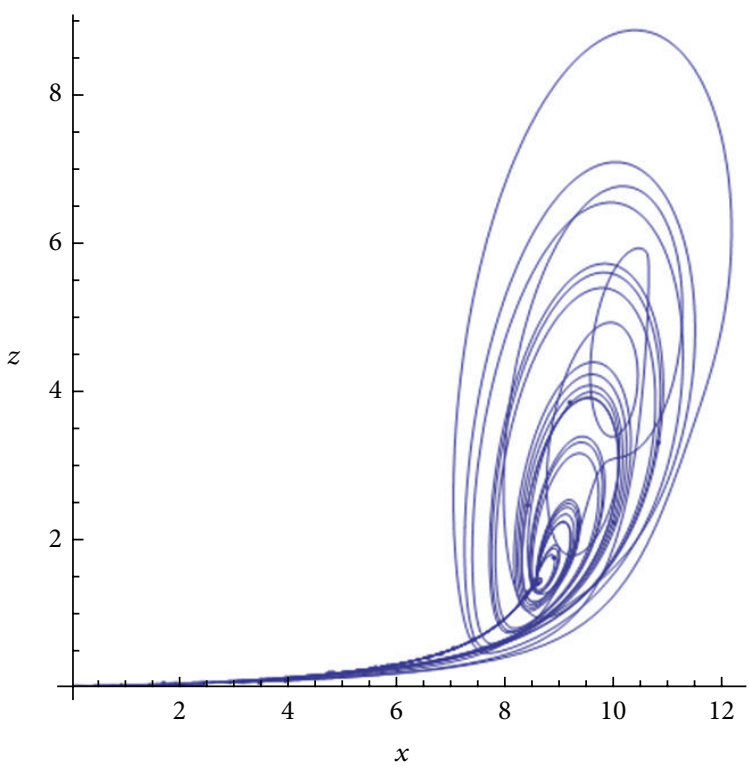

(b)

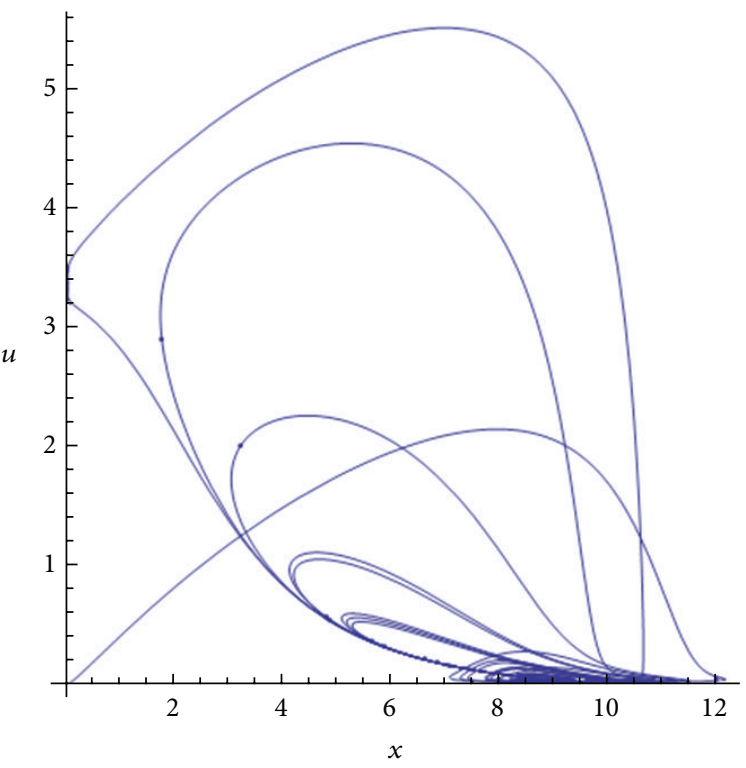

(d)

Figure 1: Phase space of $\mathrm{Ca}^{2+}$ oscillation at $q=1$.

The fractional chaotic dynamical systems have attracted increasing attention, for example, chaos in a fractional-order Rössler system [4], chaotic dynamics of fractional-order Arneodo's systems [3], fractional Chen system [5], chaos in a fractional order modified Duffing system [6], chaos in the Newton-Leipnik system with fractional order [7], fractional order Chua's system [8], fractional-order Volta's system [9], fractional model for malaria transmission under control strategies [10] and fractional order logistic [11], and discrete chaos in fractional sine and standard maps [12].

The aim of this paper is to investigate the complex dynamics of fractional-order $\mathrm{Ca}^{2+}$ oscillation model purposed by Kummer et al. [21]. The model is obtained from experimental study of hepatocyte. This mathematical model is exhibiting various dynamical behaviors, such as periodic and periodic doubling, quadrupling, and chaos. The numerical calculation of bifurcation parameters is carried out using modified trapezoidal rule for fractional integral.

\section{Role of Calcium Oscillations}

The biological system provided good examples of nonlinear dynamical system to study including the population of living things in ecosystem and biochemical oscillation and spread of epidemic diseases [19]. Oscillations in living thing are an important biological rhythmic [22]. These phenomena play an important role in many aspects of biological order and function, which involve many systems ranging from 


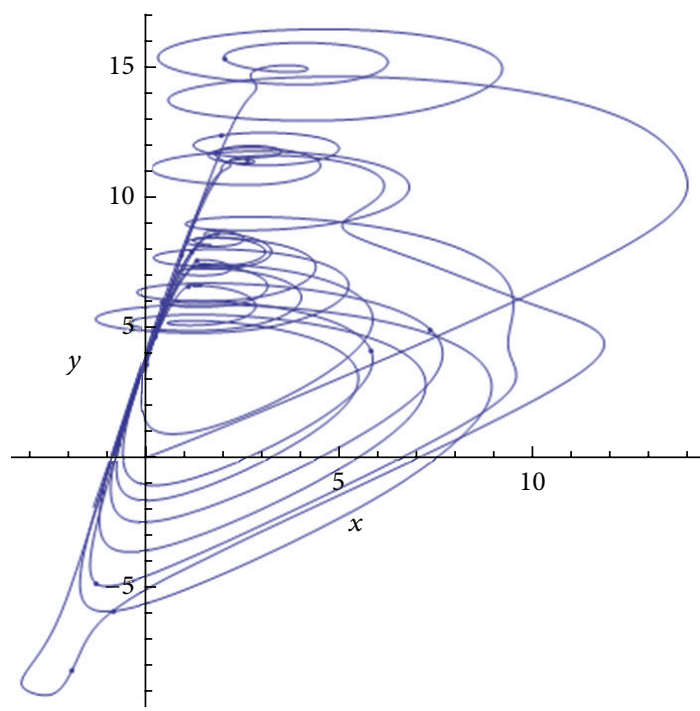

(a)

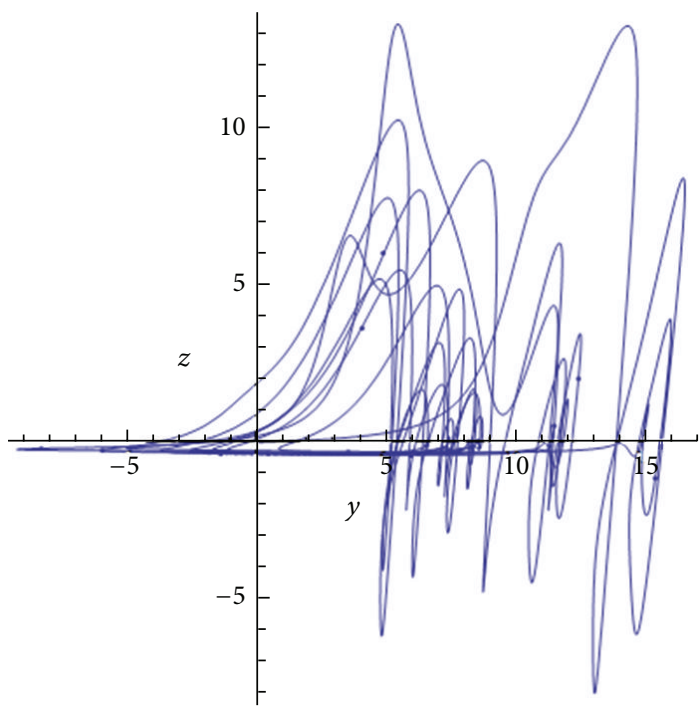

(c)

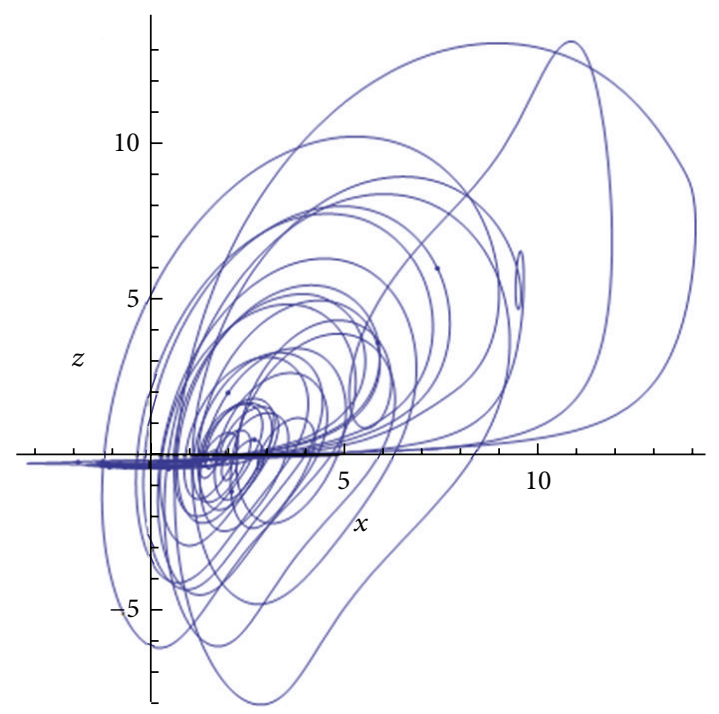

(b)

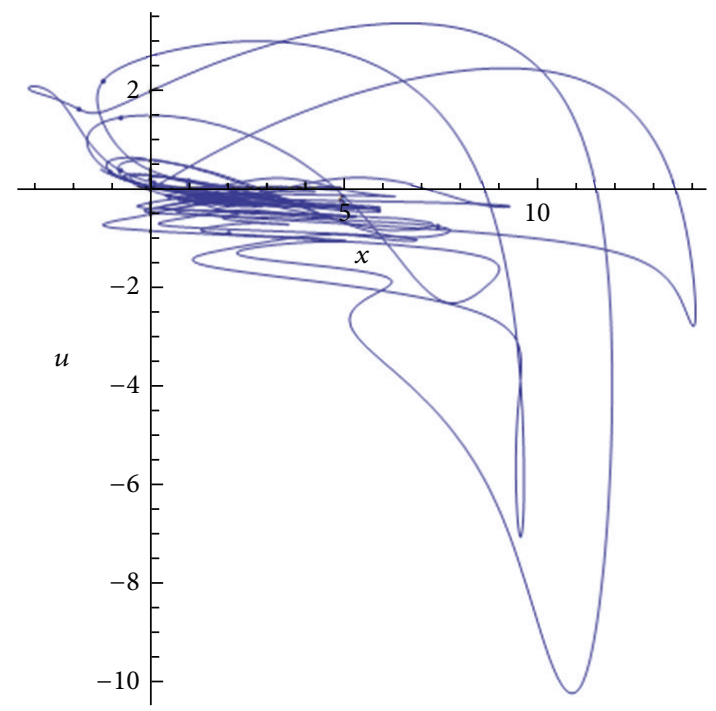

(d)

FIgURE 2: Fractional-order phase space of $\mathrm{Ca}^{2+}$ oscillation $q=0.6$.

the molecular to the macroscopic scale. The rhythmic cycles may range from microsecond to hours (molecular and cellular oscillations) or days (circadian rhythms) and even months (population growth cycles) [18]. Dynamical behaviors range from sustained autonomous oscillations with periodic cycles to periodic doubling and nonperiodic cycles or chaos [22]. Calcium oscillations are important oscillations at cellular level. It has many important roles to perform many specific functions ranging from fertilization to cell death [23]. There are many mathematical models proposed to describe the oscillatory changes on cytosolic calcium concentration in intracellular $[8,9]$. Many of experiments on different kinds of living cells are observed [21]. Most of the experimental data show simple periodic oscillations [24].

Woods et al. [25] discovered the calcium oscillations experimentally in 1986 and large numbers of cells show calcium oscillations after simulation by an extracellular agonist [21]. Later, the role in calcium signaling by inositol triphosphate $\left(\mathrm{IP}_{3}\right)$ was discovered by Berridge from studies of the control fluid secretion by an insect salivary gland [26]. The signaling pathway of $\mathrm{IP}_{3} / \mathrm{Ca}^{2+}$ has been adapted to control processes as diverse as fertilization, proliferation, cell contraction, secretion, and information processing in neuronal cells [26]. Many activities of living cells are controlled by calcium from both intracellular and extracellular sources to generate signals that transduce exogenous stimulation into physiological output [23]. The important role of calcium oscillations has been confirmed by many experimental works $[18,22,23]$.

The difference in $\mathrm{Ca}^{2+}$ oscillations frequency regulates different functions. The high frequency cytosolic $\mathrm{Ca}^{2+}$ oscillations regulate fast responses, such as synaptic transmission 


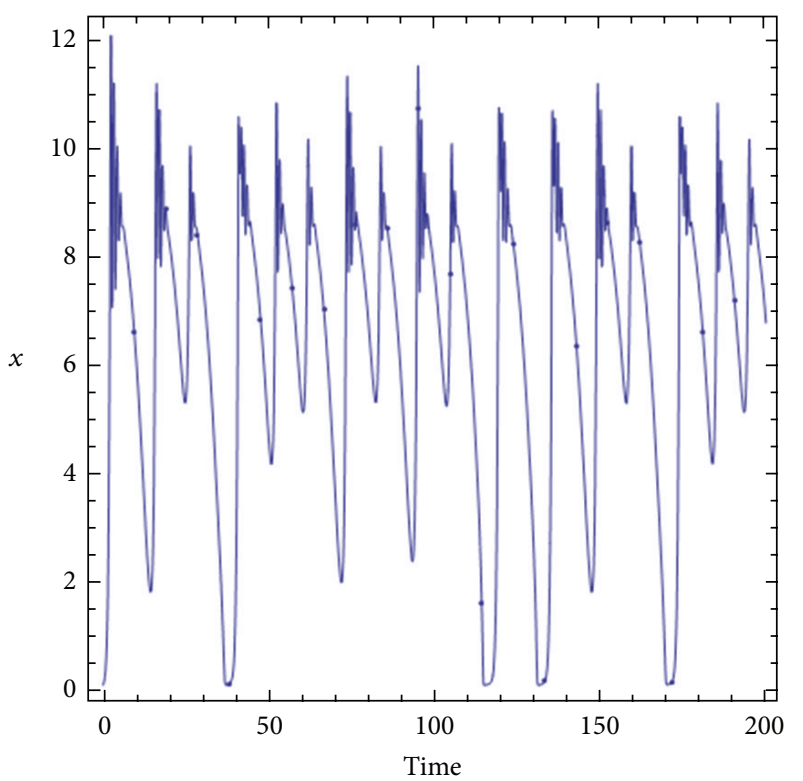

(a)

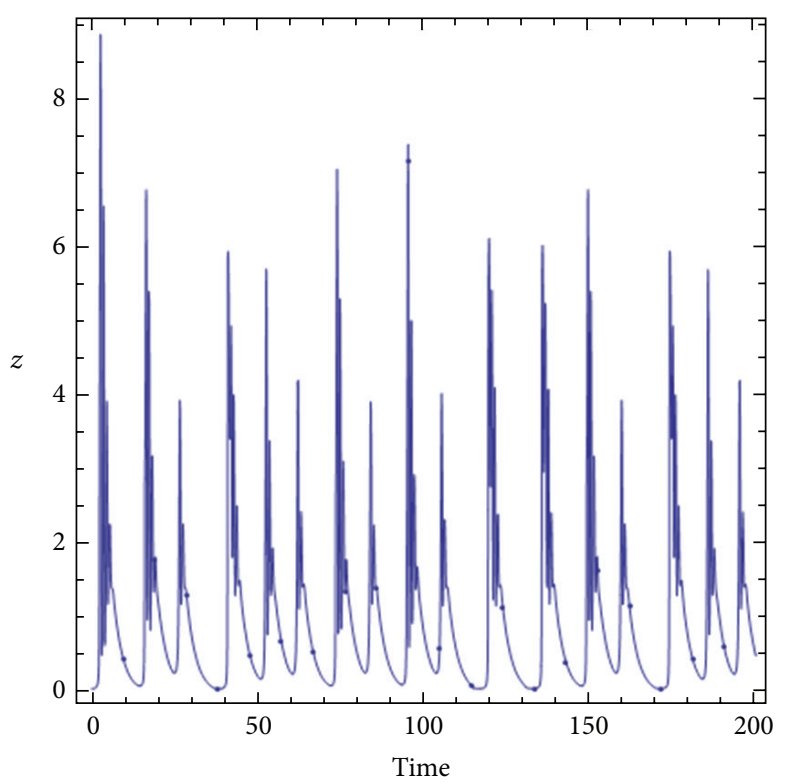

(c)

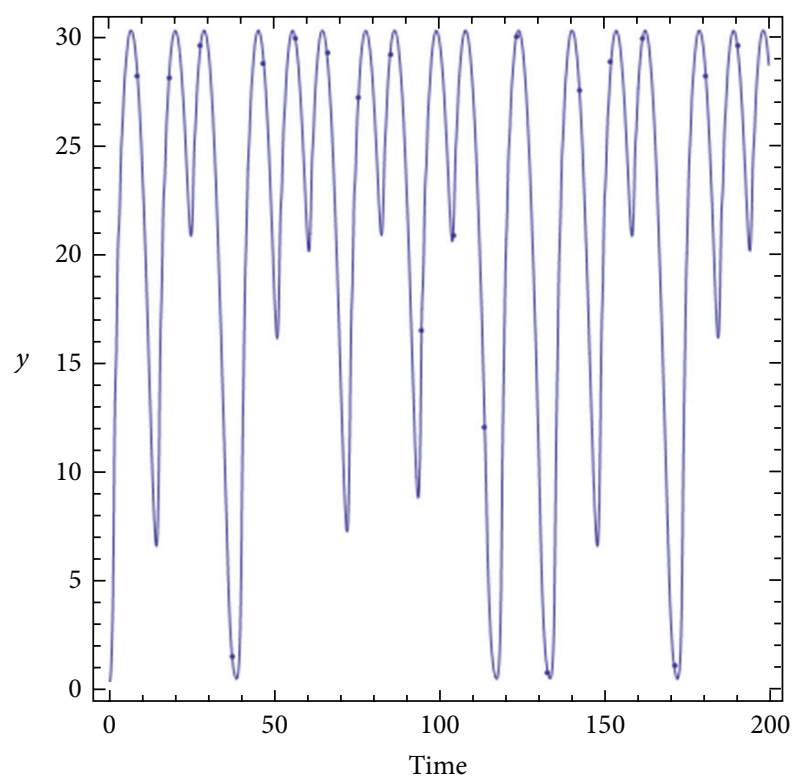

(b)

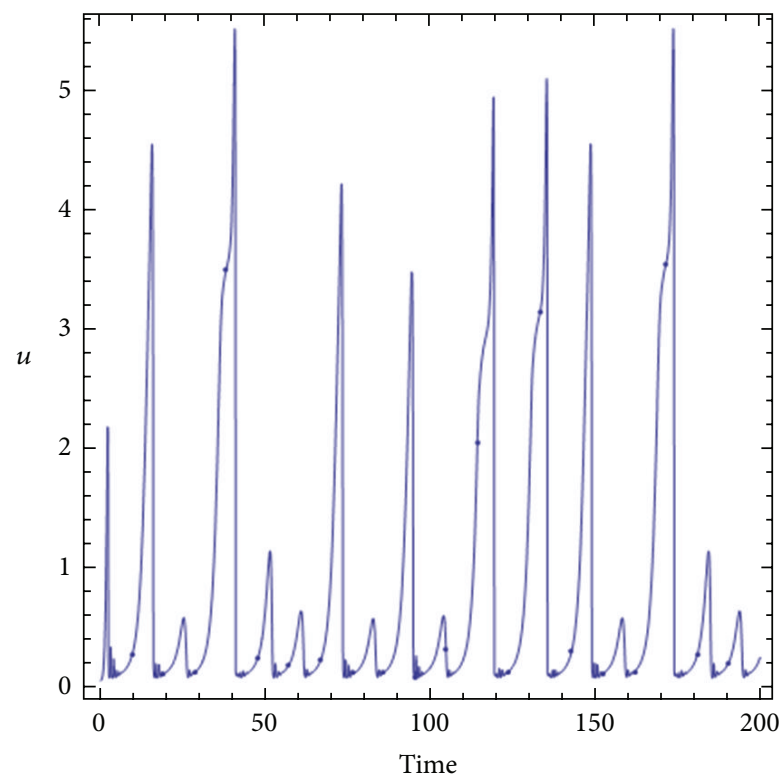

(d)

FIGURE 3: Integer-order time series with chaotic parameters.

and secretion, whereas low frequency oscillations regulate slow processes, such as fertilization and gene transcription [27]. $\mathrm{Ca}^{2+}$ oscillations have essential role in intracellular signaling [21]. The oscillating signal can have different frequency and amplitude depending upon the encoding of specific message that triggers the functions [28]. There are many mathematical models proposed to describe the mechanism of calcium oscillations but most of them present only simple periodic oscillations which are characterized by a single frequency [10].

There exist non-periodic complex bursting types of calcium oscillations found in some experimental study [21]. The models were proposed; that is, Borghans et al. [24] proposed calcium-induced calcium release (CICR) based on model focusing on the effect of cytosolic calcium on the degradation of inositol trisphosphate. Shen and Larter [29] proposed theoretical study of complex calcium oscillations model based on both the calcium-induced calcium release (CICR) and the inositol triphosphate $\left(\mathrm{IP}_{3}\right)$ cross-coupling (ICC). The model has demonstrated regular bursting and a transition to chaos which involves differential equations for cytosolic $\mathrm{Ca}^{2+}$, endoplasmic $\mathrm{Ca}^{2+}$, and $\mathrm{IP}_{3}$. Dupont and Goldbeter [30] have developed some mathematical models; one of them is related to the activation and autophosphorylation of the multifunctional $\mathrm{Ca}^{2+}$-calmodulin-dependent protein kinase II (CaMKII) by $\mathrm{Ca}^{2+}$ and calmodulin (CaM) which is able 


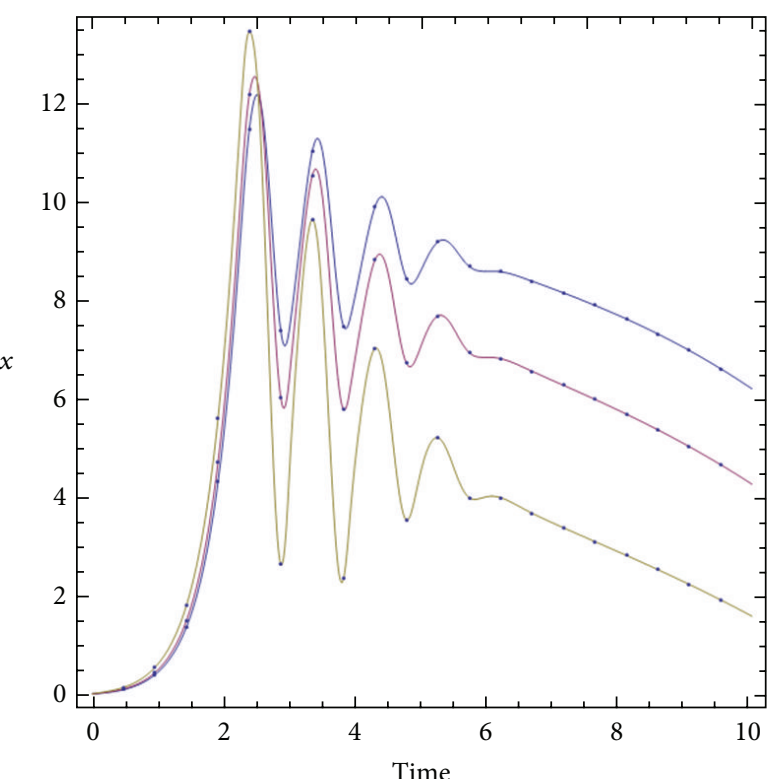

(a)

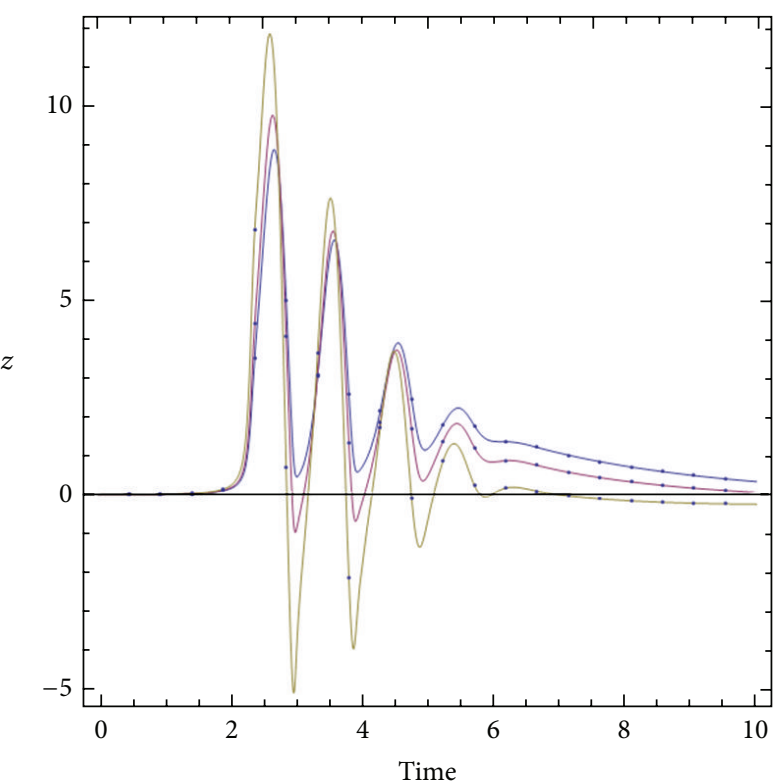

$\begin{aligned} q & =1 \\ q & =0.9 \\ q & =0.7\end{aligned}$

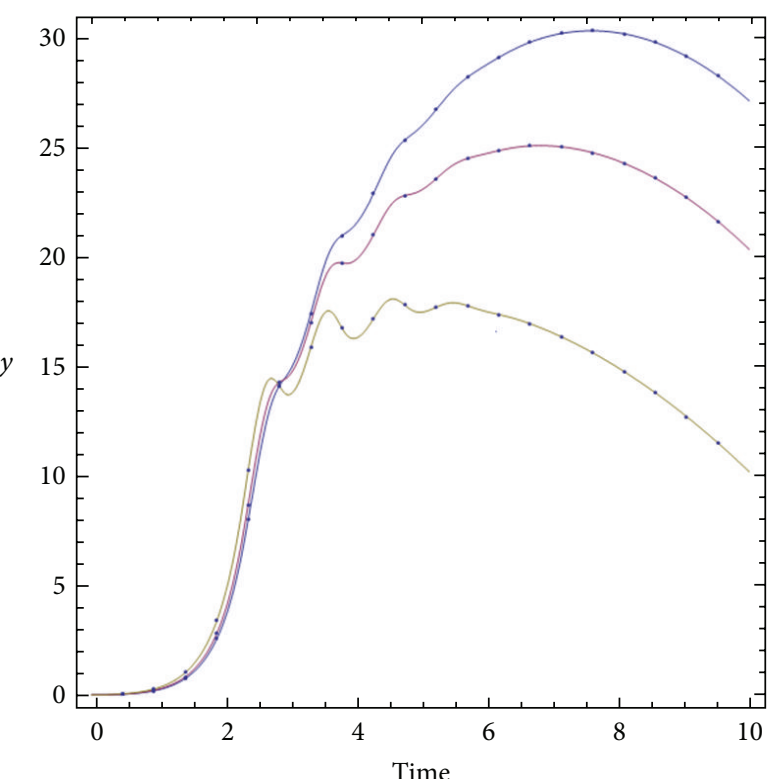

(b)

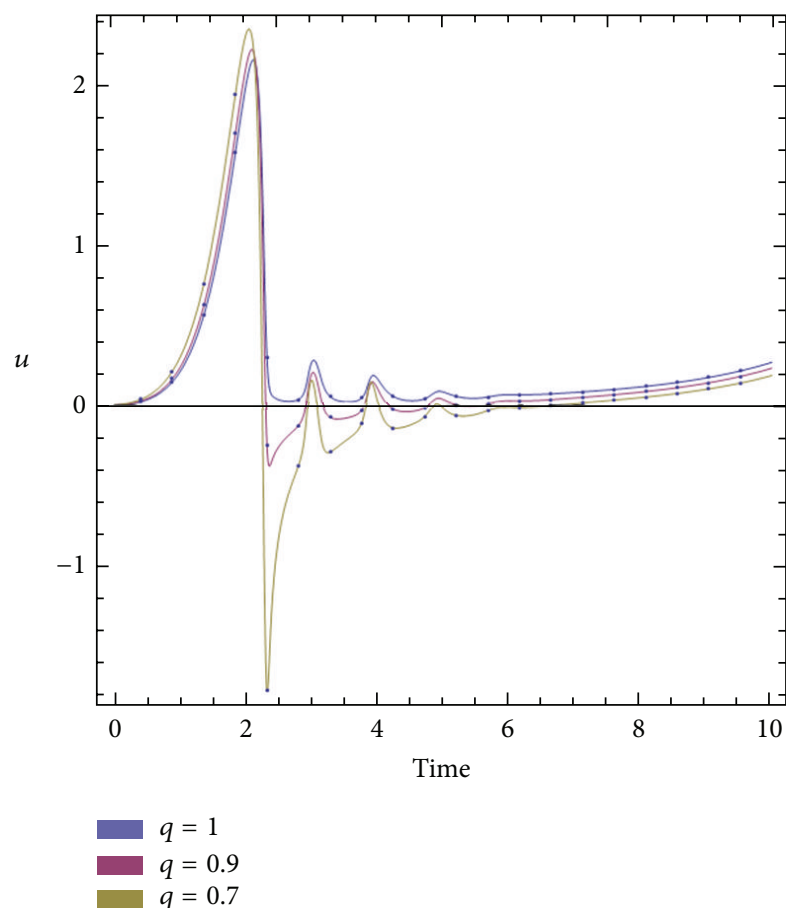

(d)

(c)

FIGURE 4: Fractional-order time series with chaotic parameters.

through underlying its ability to decode $\mathrm{Ca}^{2+}$ oscillations and to control multiple cellular functions.

Meyer and Stryer [31] proposed the inositol trisphosphate- $\left(\mathrm{IP}_{3^{-}}\right) \mathrm{Ca}^{2+}$ cross coupling model (ICC) in which the cooperative release of $\mathrm{Ca}^{2+}$ from an $\mathrm{IP}_{3}$-sensitive store results in positive feedback by $\mathrm{Ca}^{2+}$ on phospholipase C (PLC). Borghans et al. [24] proposed the calcium-induced calcium release (CICR) mechanism based on the processes originally described in skeletal triggered by calcium itself via a positive feedback loop. de Young and Keizer $\mathrm{IP}_{3} \mathrm{R}$ model [32] has one $\mathrm{IP}_{3}$ and two $\mathrm{Ca}^{2+}$ binding sites on the cytosolic side. Kummer et al. [21] proposed calcium oscillation in hepatocytes on the basis of qualitative experimental results. This mathematical model consists of the four variables as follows: cytosolic $\mathrm{Ca}^{2+}$, endoplasmic $\mathrm{Ca}^{2+}$, concentrations of active subunits of a G protein, and active PLC [21]. The model shows good agreement with experimental observations in two respects. First, each oscillation period starts with a large, steep spike 


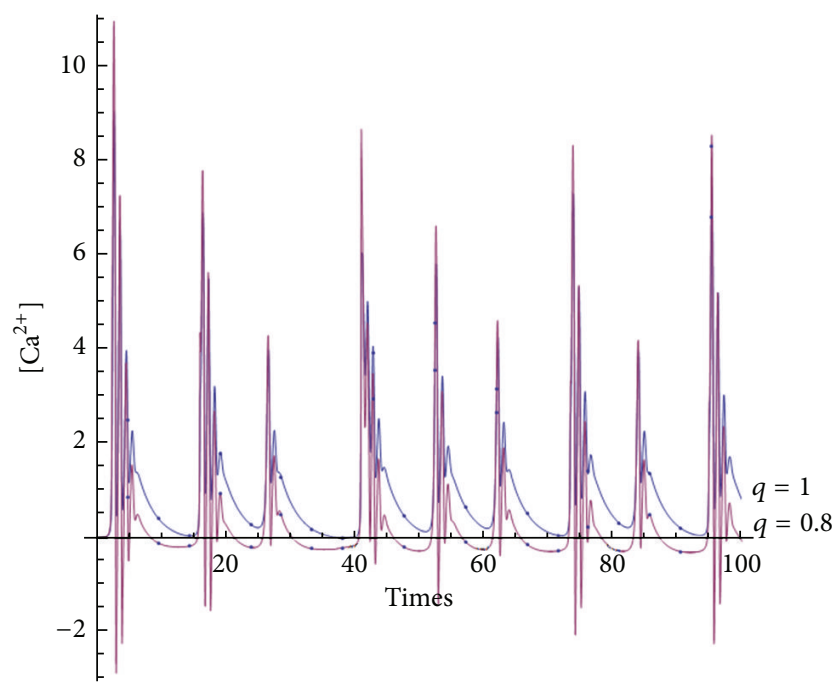

Figure 5: Fractional-order time series of $\mathrm{Ca}^{2+}$ oscillations.

followed by a number of pulses of decreasing amplitude around an elevated mean value. Second, varying the model parameters, one finds that the difference in stimulation nature can induce (periodic or aperiodic) bursting or regular oscillations [33].

Calcium has many roles for controlling numerous cellular functions. Intracellular signaling pathways are often regulated by the $\mathrm{Ca}^{2+}$ and CAMP [19]. Many physiological processes such as egg fertilization, short-term memory, neurotransmission, and gene transduction and triggering cell to death are controlled by $\mathrm{Ca}^{2+}$ oscillation [28]. The $\mathrm{Ca}^{2+}$ oscillation can be explained by change in cytosolic $\mathrm{Ca}^{2+}$ concentration.

A comprehensive review of the calcium modeling is provided by Schuster et al. [34]. Calcium has important role in information processing. It has functions as messengers. Proteins within the cell have the capacity to decode the information from the $\mathrm{Ca}^{2+}$ signals to perform different functions [19].

\section{Fractional Calculus}

Fractional calculus is the theory of differential and integral operator of non-integer order. This old mathematical topic has origin date back to the day that Leibnitz replies to L'Hospital about the meaning of half order derivative [35]. Theory is not known to scientists and engineers much until the last few decades. The theory has been applied to model many physical processes successfully with fractional differential equations including theory of viscoelasticity, heat diffusion, and nonlinear dynamics. The comprehensive discussions of this theory are presented by Oldham and Spanier [35], Miller and Ross [36], and Podlubny [37]. There are many approaches for solving the fractional-order differential equation (FDE) including series expansion, numerical approximation, Laplace transform [38], Adomian decomposition [39], predictor corrector scheme [40], Galerkin approximation [41], and Adam Moulton algorithm [42].
The applications of fractional calculus in physics are better in describing the diffusion phenomena in inhomogeneous media with non-integer derivative [37], modeling of ultracapacitors [43], the fractional derivatives model of viscoelastic material [44], thermal modeling and temperature estimation of a transistor junction [45], fractional-order impedance in electric circuit [37], dynamical process of heat conduction, and chaotic dynamical system [4-17].

In classical calculus, the meaning of integer-order derivative is the rate of change, direction of decline, or slope in the geometric interpretation. The meaning of fractional-order derivative is different. It has no obvious geometric meaning or physical interpretation [37]. Recently, Podlubny has proposed a new physical interpretation based on general convolution integrals of the Volterra type [46]. Tenreiro Machado gives a geometric and probabilistic interpretation based on Grunwald-Letnikov definition of the fractional derivative [47]. Du et al. explain physical meaning of the fractional order which is an index of memory [48]. Sabatier et al. impose physically coherent initial conditions to a fractional system [42]. Recently, this mathematical theory gains more attention in biological science, that is, low frequency constant-phase behavior in the respiratory impedance [49], front dynamics in fractional-order epidemic models [15], fractional derivatives in Dengue epidemics [14], and dynamical characteristics of the fractional-order FitzHugh-Nagumo model neuron [13].

The advantage of fractional derivatives in comparison with classical integer-order calculus is description of memory properties. In the last few decades many authors show that derivatives and integral of noninteger order are very suitable for describing properties of various real materials, for example, polymers; Bagley and Torvik show that the fractional-order models are more adequate than integerorder models [44].

\section{Definition}

Definition 1. The Riemann-Liouville fractional integral of order $q>0$ of a function $f: R^{+} \rightarrow R$ is given by

$$
I^{q} f(x)=\frac{1}{\Gamma(q)} \int_{0}^{x}(x-t)^{q-1} f(t) d t
$$

provided the right side is pointwise defined on $R^{+}$[37].

Where $\Gamma(\cdot)$ is Gamma function define by

$$
\Gamma(x)=\int_{0}^{\infty} e^{-u} u^{x-1} d u .
$$

Definition 2. The Caputo fractional derivative of order $q \in$ $(n-1, n)$ of a continuous function $f: R^{+} \rightarrow R$ is given by [37]

$$
D^{q} f(x)=I^{n-q} D^{n} f(x), \quad D=\frac{d}{d t} .
$$

Definition 3. Grunwald-Letnikov definition for fractional derivative of order $q$ is given by [37]

$$
{ }_{a} D_{t}^{q} f(t)=\lim _{h \rightarrow 0} \frac{1}{h^{q}} \sum_{j=0}^{[t-a / h]}(-1)\left(\begin{array}{l}
q \\
j
\end{array}\right) f(t-j h),
$$




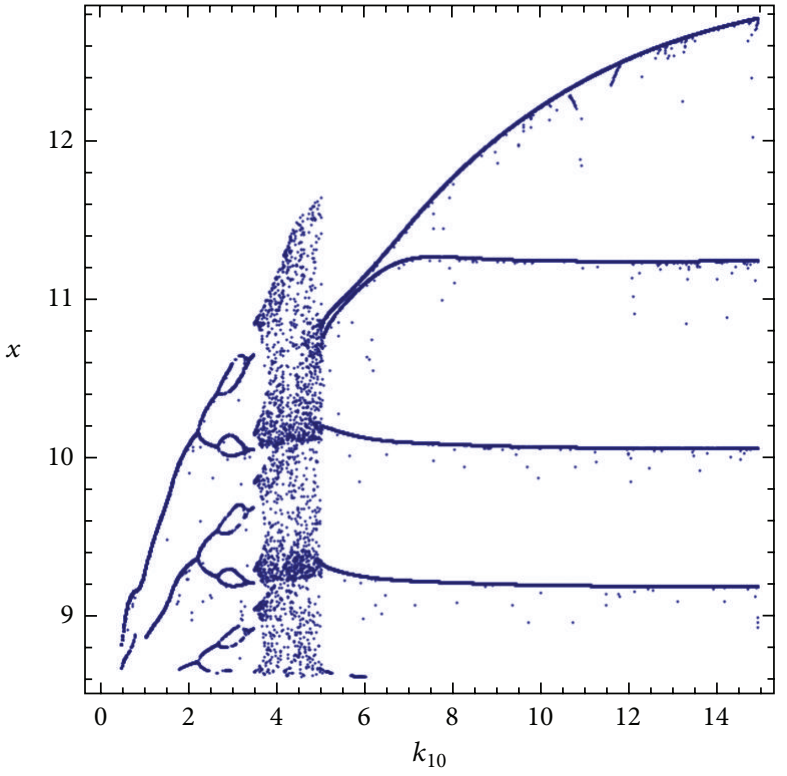

(a)

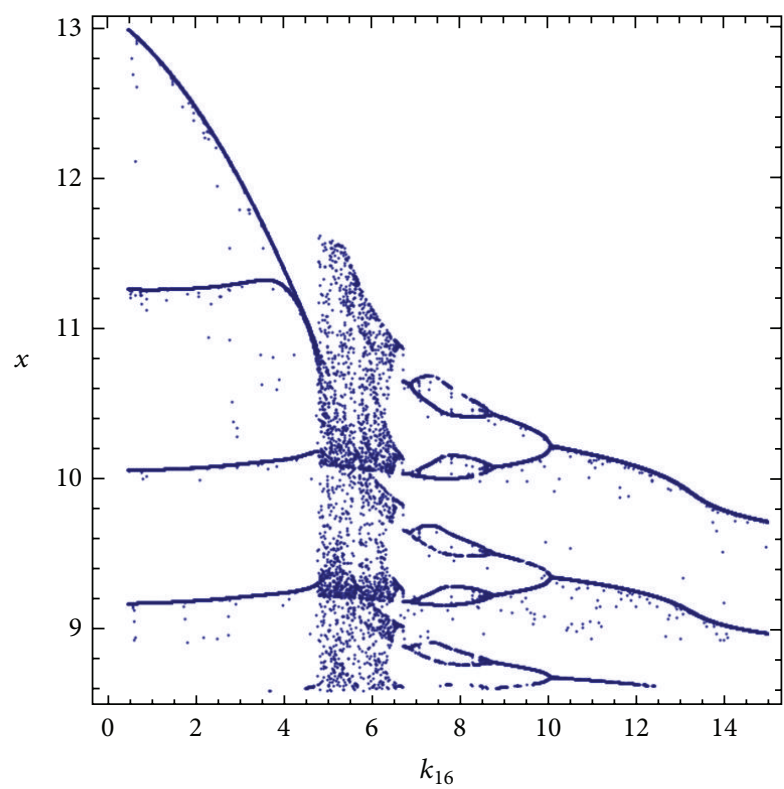

(c)

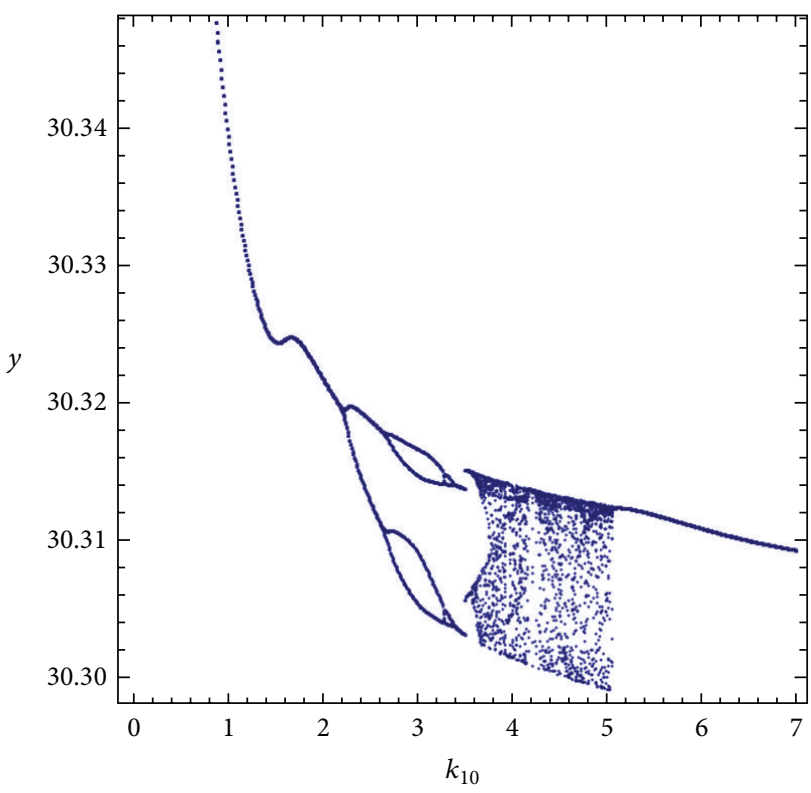

(b)

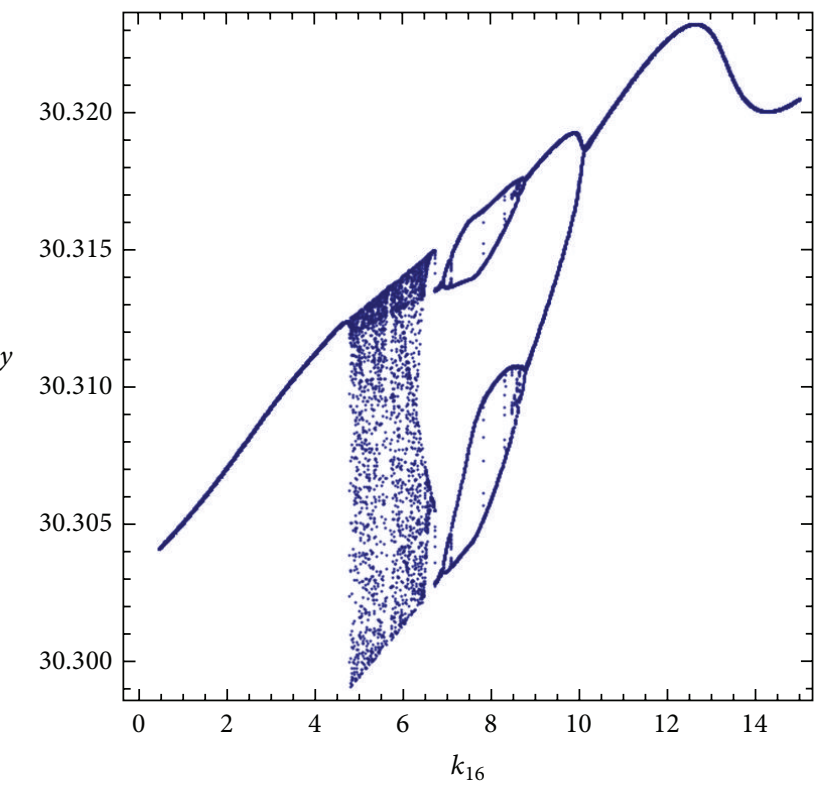

(d)

FIgURE 6: The bifurcation diagram of $\mathrm{Ca}^{2+}$ oscillations model with different control parameters.

where

$$
\begin{aligned}
\left(\begin{array}{l}
n \\
r
\end{array}\right) & =\frac{n !}{r !(n-r) !}=\frac{n(n-1)(n-2) \cdots(n-r+1)}{r !} \\
& =\frac{\Gamma(n+1)}{\Gamma(r+1) \Gamma(n-r+1)} .
\end{aligned}
$$

There are many definitions in fractional calculus. Two mostly used definitions are Riemann-Liouville and GrunwaldLetnikov. The Riemann-Liouville definition for the fractional integral and derivative is used for solving the analytical solution while the Grunwald-Letnikov definition is more appropriate in numerical calculation [35-37].

\section{Mathematical Model}

The model proposed by Kummer et al. [21] focuses on the feedback inhibition on the initial agonist receptor complex by $\mathrm{Ca}^{2+}$ and activated phospholipase $\mathrm{C}$ (PLC) and receptor type-dependent self-enhanced behavior of the activated $G_{\alpha}$ subunit. So the four main variables are the free $\mathrm{Ca}^{2+}$ concentration in the cytosol $\left(\mathrm{Ca}_{\text {cyt }}\right)$, the concentration of the active 


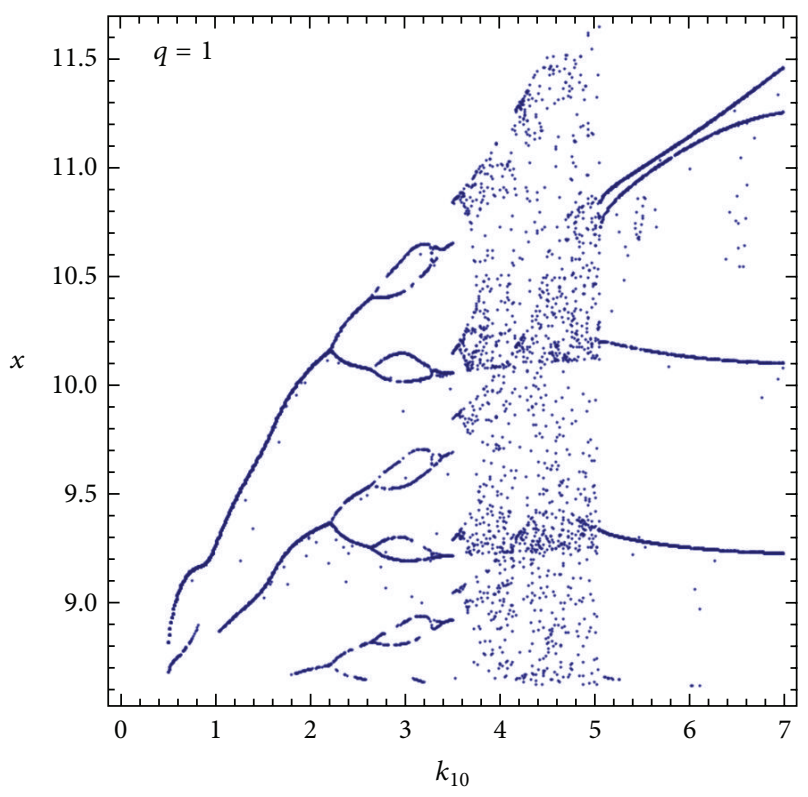

(a)

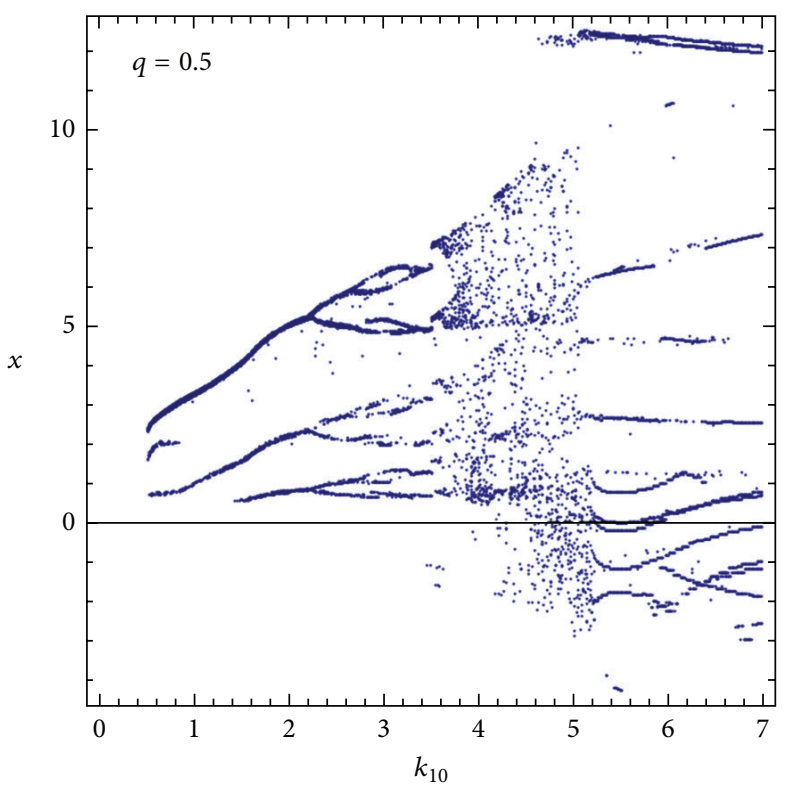

(b)

FIGURE 7: Fractional-order bifurcation of $\mathrm{Ca}^{2+}$ oscillation model.

$G_{\alpha}$ subunit, the concentration of active PLC (PLC), and the concentration of $\mathrm{Ca}^{2+}$ in endoplasmic reticulum.

Kummer et al. [21] assume that $\mathrm{IP}_{3}$ is in a quasi-stationary state where the concentration of inositol triphosphate $\left(\mathrm{IP}_{3}\right)$ follows the dynamics of active PLC. The model can be described by the four differential equations as follows:

$$
\begin{aligned}
& x^{\prime}=k_{1}+k_{2} x-k_{3} \frac{x y}{x+k_{4}}-k_{5} \frac{x z}{x+k_{6}}, \\
& y^{\prime}=k_{7} x-k_{8} \frac{y}{y+k_{9}}, \\
& z^{\prime}=k_{10} y z \frac{u}{u+k_{11}}+k_{12} y+k_{13} x-k_{14} \frac{z}{z+k_{15}}-k_{16} \frac{z}{z+k_{17}}, \\
& u^{\prime}=-k_{10} y z \frac{u}{u+k_{11}}+k_{16} \frac{z}{z+k_{17}},
\end{aligned}
$$

where

$x$ is the change in the concentration of the active $G_{\alpha}$ subunit;

$y$ is the concentration of active phospholipase C (PLC);

$z$ is the concentration of free calcium in the cytosol $\left(\mathrm{Ca}_{\text {cyt }}\right)$;

$u$ is the concentration of calcium in the intracellular stores such as endoplasmic reticulum (ER) and $k_{1}$ to $k_{17}$ are constant values.
We have used the same initial values as Kummer et al. [21] as follows:

$$
\begin{gathered}
k_{1}=0.09, \quad k_{2}=2.7738, \quad k_{3}=0.64, \\
k_{4}=0.19, \quad k_{5}=4.88, \quad k_{6}=1.18, \\
k_{7}=2.08, \quad k_{8}=32.24, \\
k_{9}=29.09, \quad k_{10}=5.0, \quad k_{11}=2.67, \quad k_{12}=0.7, \\
k_{13}=13.58, \quad k_{14}=153, \quad k_{15}=0.16, \\
k_{16}=4.85, \quad k_{17}=0.05 .
\end{gathered}
$$

\section{Fractional-Order Model}

We consider the commensurate order where the orders are all equal. The fractional-order calcium oscillation model is simply represented by replacing the integer derivative with fractional-order derivative as follows:

$$
\begin{aligned}
D^{q} x= & k_{1}+k_{2} x-k_{3} \frac{x y}{x+k_{4}}-k_{5} \frac{x z}{x+k_{6}}, \\
D^{q} y= & k_{7} x-k_{8} \frac{y}{y+k_{9}}, \\
D^{q} z= & k_{10} y z \frac{u}{u+k_{11}}+k_{12} y+k_{13} x \\
& -k_{14} \frac{z}{z+k_{15}}-k_{16} \frac{z}{z+k_{17}}, \\
D^{q} u= & -k_{10} y z \frac{u}{u+k_{11}}+k_{16} \frac{z}{z+k_{17}},
\end{aligned}
$$

where $q$ is commensurate fractional order. 


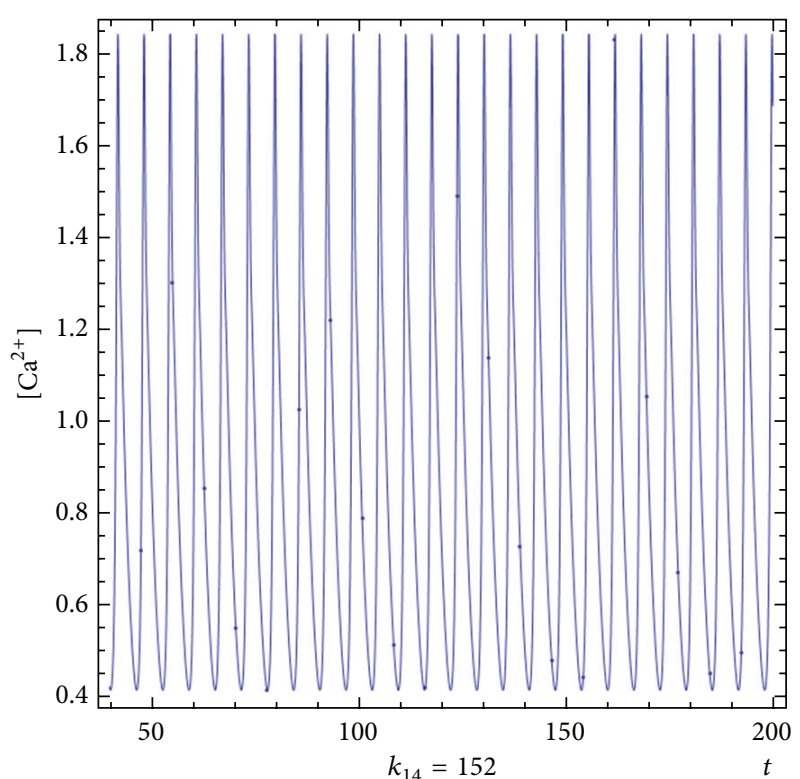

(a)

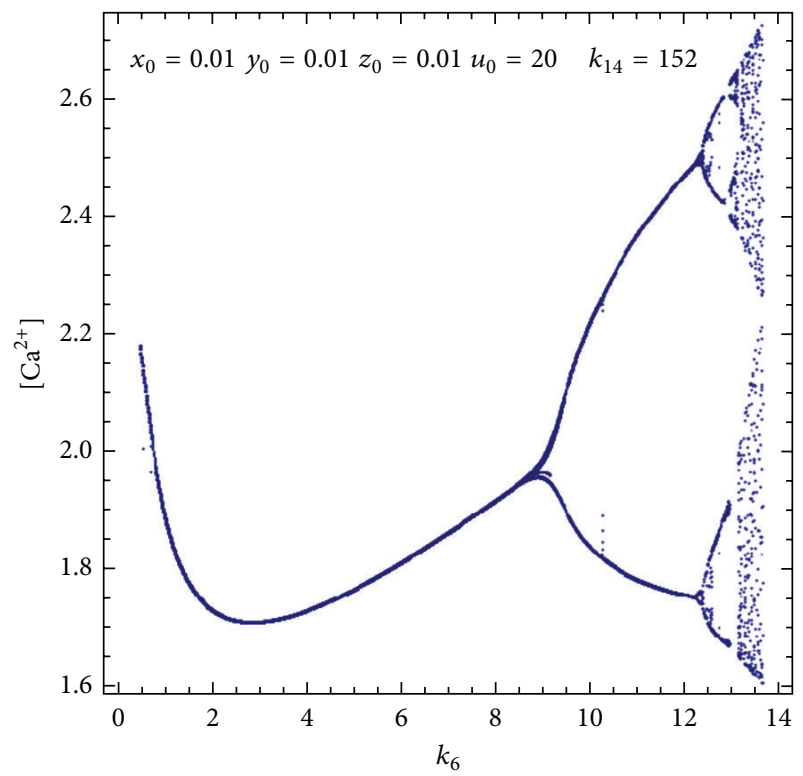

(c)

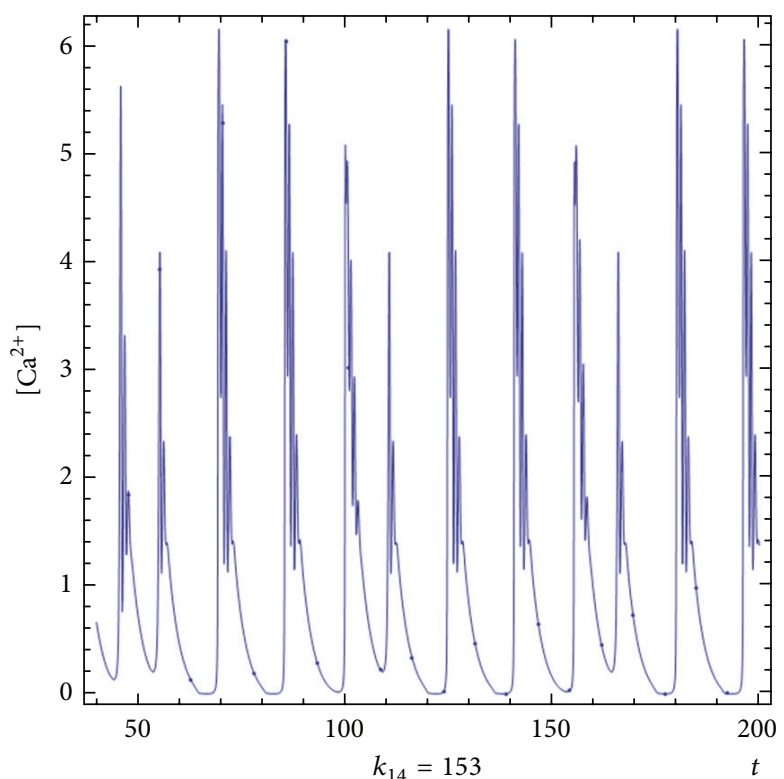

(b)

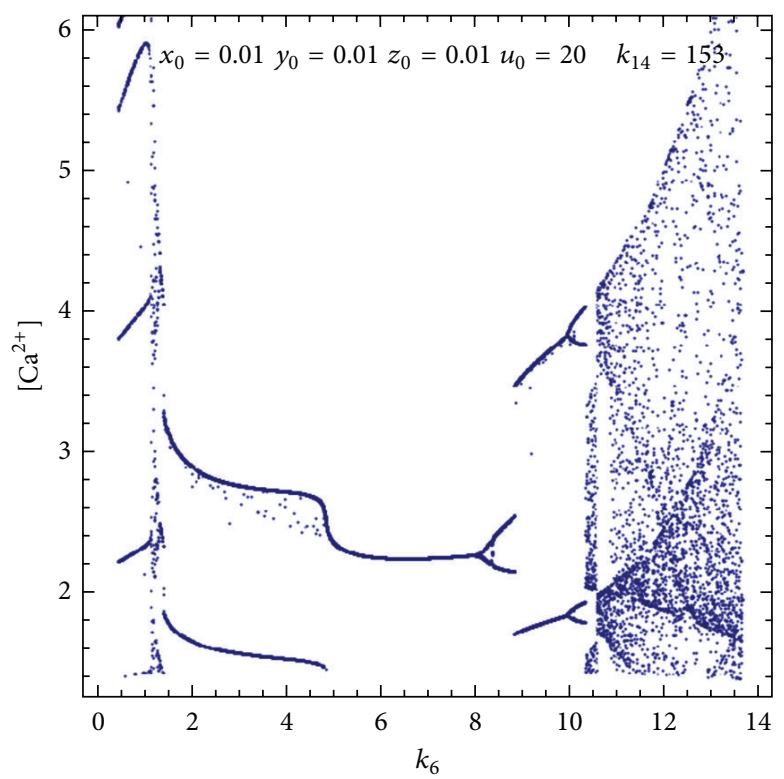

(d)

FIGURE 8: Spikes change in $\mathrm{Ca}^{2+}$ concentration for different $k_{14}$ and bifurcation diagram of $\mathrm{Ca}^{2+}$ concentration and $k_{6}$.

\section{Numerical Method}

There are many approaches in fractional-order numerical calculation. There are two main approaches for numerical calculation: the frequency domain and time domain. The study of Tavazoei and Haeri shows that the frequency domain approach can lead to the fake chaotic results [38]. The numerical method that we utilize in this paper is time domain approach of the modified trapezoidal rule proposed by Odibat and Momani [50]. This method is a simple calculation scheme that is derived from the area of trapezoidal shape.

Consider $y=f(x)$ over $[a, b]$ and suppose that the interval $[a, b]$ is subdivided into $m$ subintervals $\left\{\left[x_{k-1}, x_{k}\right]\right\}_{k=1}^{m}$ of equal width $h=(b-a) / m$ by using the equally spaced nodes $x_{k}=x_{0}+k h$ for $k=1,2, \ldots, m$.

The composite trapezoidal rule for $m$ subinterval is

$$
T(f, h)=\frac{h}{2}(f(a)+f(b))+h \sum_{k=1}^{m} f\left(x_{k}\right) .
$$


The formula can extend to use with fractional-order differential as follows [50]:

$$
\begin{aligned}
T(f, h, q)= & \left((k-1)^{q+1}-(k-q-1) k^{q}\right) \frac{h^{q} f(0)}{\Gamma(q+2)} \\
& +\sum_{j=1}^{k-1}\left((k-j+1)^{q+1}\right. \\
& \times \frac{h^{q} f\left(x_{j}\right)}{\Gamma(q+2)} .
\end{aligned}
$$

The formula in (10) is used for approximating the integral function of arbitrary order. According to the classical theory of ordinary differential equations, to produce a unique solution for the problem, we need to specify initial conditions [50]. order $q$

The equation is an approximation to fractional integral at

$$
\left(I^{q} f(x)(a)\right)=T(f, h, q)-E(f, h, q),
$$

where $a>0$ and $q>0$.

Odibat and Momani [50] show that the error is function of parameter $h$

$$
|E(f, h, q)|=O\left(h^{2}\right) .
$$

It is obvious that if the order $q=1$, then the modified trapezoidal rule reduces to the classical trapezoidal rule

$$
\lim _{q \rightarrow 1} D^{q} f(x)=\frac{d f(x)}{d x} .
$$

\section{Stability at Fractional Order}

In this section, we discuss the stability condition for fractional-order systems and a necessary condition for chaos to exist. Consider the following fractional differential equation [6]:

$$
D^{q} x_{i}=f_{i}\left(x_{1}, x_{2}, x_{3}\right), \quad i=1,2,3 .
$$

The equilibrium points $x_{i}^{\text {eq }}$ of the fractional-order differential system can be obtained by solving the following equation:

$$
D^{q} x_{i}=0
$$

and $\delta_{i}$ a small deviation can be obtained from the following equilibrium:

$$
\begin{aligned}
f_{i}\left(x_{1}^{\mathrm{eq}}+\delta_{1}(t), x_{2}^{\mathrm{eq}}+\delta_{2}(t), x_{3}^{\mathrm{eq}}+\delta_{3}(t)\right) \\
=f_{1}\left(x_{1}^{\mathrm{eq}}, x_{2}^{\mathrm{eq}}, x_{3}^{\mathrm{eq}}\right)+\left.\frac{\partial f_{1}}{\partial x_{1}}\right|_{\mathrm{eq}} \delta_{1}+\left.\frac{\partial f_{1}}{\partial x_{2}}\right|_{\mathrm{eq}} \delta_{2}+\left.\frac{\partial f_{1}}{\partial x_{3}}\right|_{\mathrm{eq}} \delta_{3},
\end{aligned}
$$

and then

$$
D^{q}\left(\delta_{i}\right) \cong+\left.\frac{\partial f_{1}}{\partial x_{1}}\right|_{\mathrm{eq}} \delta_{1}+\left.\frac{\partial f_{1}}{\partial x_{2}}\right|_{\mathrm{eq}} \delta_{2}+\left.\frac{\partial f_{1}}{\partial x_{3}}\right|_{\mathrm{eq}} \delta_{3},
$$

Therefore, we will have a linear system

$$
D^{q}\left(\delta_{i}\right)=A \delta,
$$

where

$$
A=\left[\begin{array}{lll}
\frac{\partial f_{1}}{\partial x_{1}} & \frac{\partial f_{1}}{\partial x_{2}} & \frac{\partial f_{1}}{\partial x_{3}} \\
\frac{\partial f_{2}}{\partial x_{1}} & \frac{\partial f_{2}}{\partial x_{2}} & \frac{\partial f_{2}}{\partial x_{3}} \\
\frac{\partial f_{3}}{\partial x_{1}} & \frac{\partial f_{3}}{\partial x_{2}} & \frac{\partial f_{3}}{\partial x_{3}}
\end{array}\right]
$$

The following autonomous system:

$$
D^{q} x=A x, \quad x(0)=x_{0},
$$

where $0<q<1, x \in R^{n}$, and $A \in R^{n \times n}$, is asymptotically stable if and only if $\left|\arg \left(\lambda_{i}(A)\right)\right|>q \pi / 2$. In this case, each component of the states decays towards 0 like $t^{-q}$ Also; this system is stable if and only if $\left|\arg \left(\lambda_{i}(A)\right)\right| \geq q \pi / 2$ and those critical eigenvalues that satisfy $\left|\arg \left(\lambda_{i}(A)\right)\right|=q \pi / 2$ have geometric multiplicity one [17].

\section{Commensurate Fractional Order}

Consider that the commensurate fractional-order system with the order is all equal to $q$. In this case, a system shows regular behavior if it satisfies [51]

$$
q<\frac{2}{\pi} \min _{i}\left|\arg \left(\lambda_{i}\right)\right|
$$

The equilibrium points of the system at the given parameters set are as follows:

$$
\begin{gathered}
E_{1}(10.0544,53.7091,-1.3183,-0.0374864), \\
E_{2}(-1.24025,-2.15522,-0.0171356,-2.4883), \\
E_{3}(-0.352276,-0.64645,-0.00529478,-2.59275), \\
E_{4}(-0.0299949,-0.0561849,-0.000465736,-2.66236), \\
E_{5}(7.81412,29.5754,0.775014,0.110537), \\
E_{6}(19.1242,-153.502,32.7238,-0.000514706) .
\end{gathered}
$$

From the initial condition in [10],

$$
\begin{gathered}
k_{1}=0.09, \quad k_{2}=2.7738, \quad k_{3}=0.64, \quad k_{4}=0.19, \\
k_{5}=4.88, \quad k_{6}=1.18, \quad k_{7}=2.08, \quad k_{8}=32.24, \\
k_{9}=29.09, \quad k_{10}=5.0, \quad k_{11}=2.67, \quad k_{12}=0.7, \\
k_{13}=13.58, \quad k_{14}=153, \\
k_{15}=0.16, \quad k_{16}=4.85, \quad k_{17}=0.05 .
\end{gathered}
$$


The Jacobian determinant is

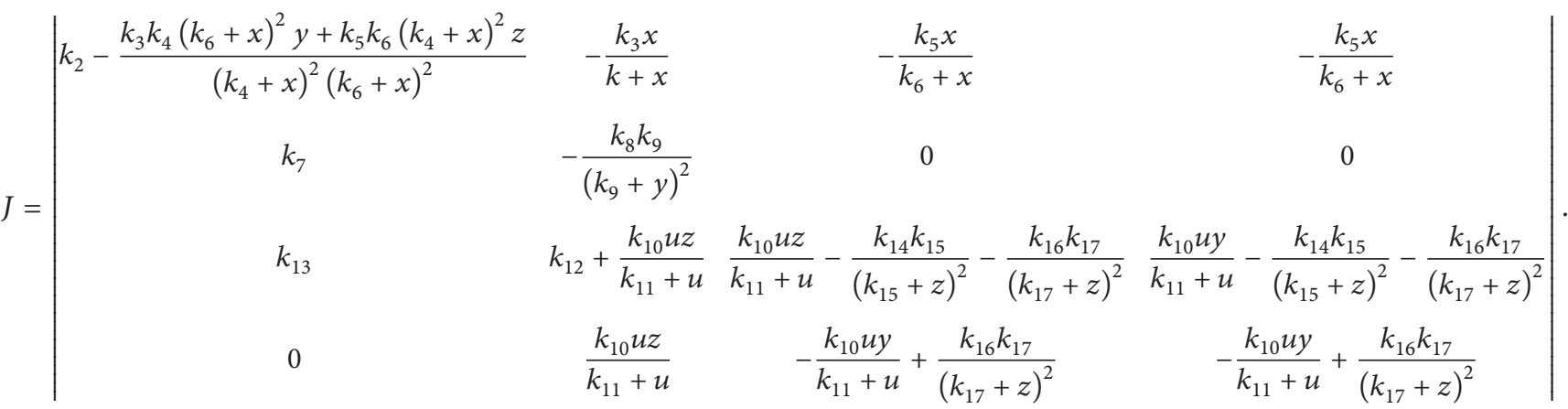

The characteristic equation is

$$
\begin{gathered}
\lambda^{4}+15.6113 \lambda^{3}+12.1598 \lambda^{2}+31.393 \lambda-4.07004 \times 10^{-16} \\
\operatorname{Arg}\left(\lambda_{1}\right)=\pi, \quad \operatorname{Arg}\left(\lambda_{2}\right)=-1.80517, \\
\operatorname{Arg}\left(\lambda_{3}\right)=1.80517, \quad \operatorname{Arg}\left(\lambda_{4}\right)=0 .
\end{gathered}
$$

From (21) system shows regular behavior if it satisfies $q<$ 1.14921 .

We have calculated the values of each argument that correspond to the equilibrium point's results in either 0 or $\pi$ which means that all equilibrium points are unstable.

\section{Fractional-Order Phase Space}

The constructing fractional-order phase space can assist in understanding the fractional change in dynamical system. Chaotic behavior of the calcium oscillation in liver cell can be viewed in phase space of the calcium concentration and other system variables. The geometric meaning of the single closed loop of phase space represents the periodic system, two closed loops of phase space represent the periodic doubling, and so on [49]. For chaotic system, the phase space appears as a multiple loop. According to the given values of the constants chaotic parameters $k_{1}$ to $k_{17}$, the phase spaces of Kummer model are presented in Figures 1 and 2, respectively.

\section{Fractional-Order Time Series}

Nonlinear dynamics are time dependent system. The change from periodic behavior to periodic doubling and then route to chaos can be analyzed from the time series. Time series represent are sequence of points which describe the behavior of dynamical system. The calcium oscillation model is characterized by four different variables: the change in the concentration of the active $G_{\alpha}$ subunit $(x)$, the concentration of active phospholipase C (PLC) $(y)$, concentration of free calcium in the cytosol $\left(\mathrm{Ca}_{\text {cyt }}\right)(z)$, and concentration of calcium in the intracellular stores $(u)$. The integer-order time series of the $\mathrm{Ca}^{2+}$ model are shown in Figure 3 and the numerical results of fractional-order time series obtained by numerical integration of the modified trapezoidal rule are shown in Figures 4 and 5, respectively. The complexities at fractional-order are present as the bursting of the $\mathrm{Ca}^{2+}$ signal.

\section{Fractional-Order Bifurcation Diagram}

Generally, the bifurcation diagram represents the behavior of dynamical system when varying certain parameter values. Single lines represent the stable periodic system, while branching represents the periodic doubling or quadrupling or higher. The band of dots represents the chaotic region. The integer-order bifurcation diagram of the model for parameter $k_{10}$ and $k_{16}$ is presented in Figure 6. The comparison between integer-order bifurcation diagram and fractional order bifurcation of the parameter $k_{10}$ is presented in Figure 7. The numerical result shows the existence of chaos at fractional order for the same control parameter and initial condition. The two diagrams look resemble but slightly different in scales.

\section{Numerical Results}

The model proposed by Kummer et al. [10] show that there exists chaotic behavior at the given parameters. We have examined further different ranges with fractional calculus for the parameters that show periodic doubling and route to chaos. The corresponding bursting fractional-order time series are also present. The numerical integration at fractional order is accomplished by applying the modified trapezoidal rule to the fractional differential equation. The numerical errors are related to the time step of numerical integration. To reduce the numerical errors, the time step is set to $h=3000$.

The numerical results with fractional order are provided by huge variations in the number of solutions. This would be able to fit to the experimental data. According to the literature, the functions of calcium are in numerous areas ranging from fertilization and muscle movement. The variation in solutions outcome would be feasible to describe certain functions caused from the oscillations. 
We have chosen the order $q=0.8$ to examine the fractional-order time series of the $\mathrm{Ca}^{2+}$ concentration as presented in Figure 5. The constant values are as follows:

$$
\begin{gathered}
k_{1}=0.09, \quad k_{2}=2.7738, \quad k_{3}=0.64, \quad k_{4}=0.19, \\
k_{5}=4.88, \quad k_{6}=1.18, \quad k_{7}=2.08, \quad k_{8}=32.24, \\
k_{9}=29.09, \quad k_{10}=5.0, \quad k_{11}=2.67, \\
k_{12}=0.7, \quad k_{13}=13.58, \quad k_{14}=153, \\
k_{15}=0.16, \quad k_{16}=4.85, \quad k_{17}=0.05 .
\end{gathered}
$$

The initial values are $x_{0}=0.01, y_{0}=0.01, z_{0}=0.01$, and $u_{0}=20$.

The bifurcation diagrams are obtained by varying certain control parameter and take the highest values from iteration. The constant values in the model that show the periodic doubling and route to chaos are $k_{6}, k_{10}, k_{11}$, and $k_{16}$.

We choose the parameter $k_{10}$ to study the fractional-order bifurcation in Figure 7 . The integer-order shows that there exist period-3 $\left(k_{10}<2.2\right)$, period-6 $\left(2.2<k_{10}<2.6\right)$, and period-10 $\left(2.6<k_{10}<3.4\right)$ and then route to chaos $(3.6<$ $\left.k_{10}<5\right)$. The fractional-order bifurcation diagram is slightly different and there still exists the chaotic region at different scale.

\section{Discussion and Conclusions}

We have shown that cytosolic $\mathrm{Ca}^{2+}$ oscillations can change the concentration very rapidly when the constant variable $k_{14}$ changes from 152 to 153 as present with time series and bifurcation diagram in Figure 8 . There are many mathematical models proposed to explain the calcium oscillations. Kummer's model of calcium oscillations in hepatocytes is able to display simple oscillatory and chaotic bursting. The slight change in certain variable can cause the abruptly change in $\mathrm{Ca}^{2+}$ concentration.

The Poincare-Bendixson theorem states that continuous dynamical systems cannot exhibit chaotic attractor if dimension is less than three. We have presented the example of biological model of $\mathrm{Ca}^{2+}$ oscillation that exhibits chaotic behavior at order less than three. In this paper, we have applied the theory of fractional calculus to study chaotic property in mathematical model of calcium oscillation proposed by Kummer et al. There exists the irregularity behavior in the model. The complex behaviors are exhibited as bursting during the oscillation. The fractional-order time series of bursting signal are present. The bifurcation diagrams show that there exists chaos with the order less than three. In conclusion, the numerical results are able to yield insight into an intermediate change in fractional order numerical integration. The variation of the solutions by fractional order integration would be feasible to describe certain functions of the $\mathrm{Ca}^{2+}$ oscillations when compared with the experimental data.

\section{Conflict of Interests}

The authors declare that there is no conflict of interests regarding the publication of this paper.

\section{References}

[1] E. N. Lorenz, "Deterministic non-periodic flow," Journal of the Atmospheric Sciences, vol. 20, pp. 130-141, 1963.

[2] S. H. Strogatz, Nonlinear Dynamics and Chaos, Westview, 2000.

[3] J. G. Lu, "Chaotic dynamics and synchronization of fractionalorder Arneodo's systems," Chaos, Solitons and Fractals, vol. 26, no. 4, pp. 1125-1133, 2005.

[4] W. Zhang, S. Zhou, H. Li, and H. Zhu, "Chaos in a fractional order Rössler system," Chaos, Solitons and Fractals, vol. 42, no. 3, pp. 1684-1691, 2009.

[5] J. G. Lu and G. Chen, "A note on the fractional-order Chen system," Chaos, Solitons and Fractals, vol. 27, no. 3, pp. 685-688, 2006.

[6] Z.-M. Ge and C.-Y. Ou, "Chaos in a fractional order modified Duffing system," Chaos, Solitons and Fractals, vol. 34, no. 2, pp. 262-291, 2007.

[7] X. Wang and L. Tian, "Bifurcation analysis and linear control of the Newton-Leipnik system," Chaos, Solitons and Fractals, vol. 27, no. 1, pp. 31-38, 2006.

[8] I. Petráš, "A note on the fractional-order Chua’s system," Chaos, Solitons and Fractals, vol. 38, no. 1, pp. 140-147, 2008.

[9] I. Petráš, "A note on the fractional-order Volta’s system," Communications in Nonlinear Science and Numerical Simulation, vol. 15, no. 2, pp. 384-393, 2010.

[10] C. M. A. Pinto and J. A. T. Machado, "Fractional model for malaria transmission under control strategies," Computers \& Mathematics with Applications, vol. 66, no. 5, pp. 908-916, 2013.

[11] Y. Suansook and K. Paithoonwattanakij, "Dynamic of logistic model at fractional order," in Proceeding of the International Symposium on Industrial Electronics (IEEE ISIE '09), pp. 718723, Seoul, Republic Korea, July 2009.

[12] G.-C. Wu, D. Baleanu, and S.-D. Zeng, "Discrete chaos in fractional sine and standard maps," Physics Letters A, vol. 378, no. 5-6, pp. 484-487, 2014.

[13] Y. Liu, Y. Xie, Y. Kang et al., "Dynamical characteristics of the fractional-order fitzHugh-Nagumo model neuron," in Advances in Cognitive Neurodynamics (II), pp. 253-258, 2011.

[14] S. Pooseh, H. S. Rodrigues, and D. F. M. Torres, "Fractional derivatives in dengue epidemics," in Proceedings of the International Conference on Numerical Analysis and Applied Mathematics: Numerical Analysis and Applied Mathematics (ICNAAM '11), vol. 1389, pp. 739-742, September 2011.

[15] E. Hanert, E. Schumacher, and E. Deleersnijder, "Front dynamics in fractional-order epidemic models," Journal of Theoretical Biology, vol. 279, pp. 9-16, 2011.

[16] V. Daftardar-Gejji and S. Bhalekar, "Chaos in fractional ordered Liu system," Computers and Mathematics with Applications, pp. 1117-1127, 2010.

[17] I. Petráš, "Method for simulation of the fractional order chaotic system," Acta Montanistica Slovaca, vol. 11, pp. 273-277, 2006.

[18] A. Goldbeter, Biochemical Oscillations and Cellular Rhythms: The Molecular Bases of Periodic and Chaotic Behavior, Cambridge University Press, 1996. 
[19] M. Falcke and D. Malchow, Understanding Calcium Dynamics: Experiments and Theory, vol. 623 of Lecture Notes in Physics, 2003.

[20] M. J. Berridge, "Calcium oscillations," The Journal of Biological Chemistry, vol. 265, no. 17, pp. 9583-9586, 1990.

[21] U. Kummer, L. F. Olsen, C. J. Dixon, A. K. Green, E. BornbergBauer, and G. Baier, "Switching from simple to complex oscillations in calcium signaling," Biophysical Journal, vol. 79, no. 3, pp. 1188-1195, 2000.

[22] A. Goldbeter, "Computational approaches to cellular rhythms," Nature, vol. 420, no. 6912, pp. 238-245, 2002.

[23] M. D. Bootman, M. J. Berridge, and H. L. Roderick, "Calcium signalling: more messengers, more channels, more complexity," Current Biology, vol. 12, no. 16, pp. R563-R565, 2002.

[24] J. A. M. Borghans, G. Dupont, and A. Goldbeter, "Complex intracellular calcium oscillations. A theoretical exploration of possible mechanisms," Biophysical Chemistry, vol. 66, no. 1, pp. 25-41, 1997.

[25] N. M. Woods, K. S. R. Kuthbertson, and P. H. Cobbold, "Agonist-induced oscillations in hepatocytes," Cells Calcium, vol. 8, pp. 79-100, 1987.

[26] M. J. Berridge, "Unlocking the secrets of cell signaling," Annual Review of Physiology, vol. 67, pp. 1-21, 2005.

[27] P. Uhlén and N. Fritz, "Biochemistry of calcium oscillations," Biochemical and Biophysical Research Communications, vol.396, no. 1, pp. 28-32, 2010.

[28] M. Perc and M. Marhl, "Sensitivity and flexibility of regular and chaotic calcium oscillations," Biophysical Chemistry, vol. 104, no. 2, pp. 509-522, 2003.

[29] P. Shen and R. Larter, "Chaos in intracellular $\mathrm{Ca}^{2+}$ oscillations in a new model for non-excitable cells," Cell Calcium, vol. 17, no. 3, pp. 225-232, 1995.

[30] G. Dupont and A. Goldbeter, "One-pool model for $\mathrm{Ca}^{2+}$ oscillations involving $\mathrm{Ca}^{2+}$ and inositol 1,4,5-trisphosphate as co-agonists for $\mathrm{Ca}^{2+}$ release," Cell Calcium, vol. 14, no. 4, pp. 311322, 1993.

[31] T. Meyer and L. Stryer, "Transient calcium release induced by successive increments of inositol 1,4,5-trisphosphate," Proceedings of the National Academy of Sciences of the United States of America, vol. 87, no. 10, pp. 3841-3845, 1990.

[32] G. W. de Young and J. Keizer, "A single-pool inositol 1,4,5trisphosphate-receptor-based model for agonist-stimulated oscillations in $\mathrm{Ca}^{2+}$ concentration," Proceedings of the National Academy of Sciences of the United States of America, vol. 89, no. 20, pp. 9895-9899, 1992.

[33] Y. Timofeeva, Oscillations and waves in single and multicellular system with free calcium [Ph.D. thesis], Loughborough University, 2003.

[34] S. Schuster, M. Marhl, and T. Höfer, "Modelling of simple and complex calcium oscillations from single-cell responses to intercellular signalling," European Journal of Biochemistry, vol. 269, no. 5, pp. 1333-1355, 2002.

[35] K. B. Oldham and J. Spanier, The Fractional Calculus, Academic Press, New York, NY, USA, 1974.

[36] K. S. Miller and B. Ross, An Introduction to the Fractional Calculus and Fractional Differential Equations, John Wiley \& Sons, New York, NY, USA, 1993.

[37] I. Podlubny, Fractional Differential Equations, vol. 198, Academic Press, San Diego, Calif, USA, 1999.

[38] M. S. Tavazoei and M. Haeri, "Limitations of frequency domain approximation for detecting chaos in fractional order systems,"
Nonlinear Analysis: Theory, Methods \& Applications, vol. 69, no. 4, pp. 1299-1320, 2008.

[39] V. Daftardar-Gejji and H. Jafari, "Adomian decomposition: a tool for solving a system of fractional differential equations," Journal of Mathematical Analysis and Applications, vol. 301, no. 2, pp. 508-518, 2005.

[40] J. Sabatier, O. P. Agrawal, and J. A. T. Machado, Advances in Fractional Calculus: Theoretical Developments and Applications in Physics and Engineering, Springer, Dordrecht, The Netherlands, 2007.

[41] S. J. Singh and A. Chatterjee, "Three classes of FDEs amenable to approximation using a Galerkin technique," in Advances in Fractional Calculus, Springer, 2007.

[42] J. Sabatier, M. Merveillaut, R. Malti, and A. Oustaloup, "How to impose physically coherent initial conditions to a fractional system?" Communications in Nonlinear Science and Numerical Simulation, vol. 15, no. 5, pp. 1318-1326, 2010.

[43] N. Bertrand, J. Sabatier, O. Briat, and J.-M. Vinassa, "Fractional non-linear modelling of ultracapacitors," Communications in Nonlinear Science and Numerical Simulation, vol. 15, no. 5, pp. 1327-1337, 2010.

[44] R. L. Bagley and P. J. Torvik, "A theoretical basis for the application of fractional calculus to viscoelasticity," Journal of Rheology, vol. 27, no. 3, pp. 201-210, 1983.

[45] J. Sabatier, H. C. Nguyen, C. Farges et al., "Fractional models for thermal modeling and temperature estimation of a transistor junction," Advances in Difference Equations, vol. 201, Article ID 687363, 12 pages, 2011.

[46] I. Podlubny, "Geometric and physical interpretation of fractional integration and fractional differentiation," Fractional Calculus \& Applied Analysis, vol. 5, no. 4, pp. 367-386, 2002.

[47] J. A. Tenreiro Machado, "A Probabilistic Interpretation of the fractional-order differentiation," Fractional Calculus \& Applied Analysis, vol. 6, no. 1, pp. 73-80, 2003.

[48] M. Du, Z. Wang, and H. Hu, "Measuring memory with the order of fractional derivative," Scientific Reports, vol. 3, article 3431, 2013.

[49] C. Ionescu, R. de Keyser, J. Sabatier, A. Oustaloup, and F. Levron, "Low frequency constant-phase behavior in the respiratory impedance," Biomedical Signal Processing and Control, vol. 6, no. 2, pp. 197-208, 2011.

[50] Z. Odibat and S. Momani, "An Algorithm for the numerical solution of differential equations of fractional order," Journal of Applied Mathematics \& Informatics, vol. 26, no. 1-2, pp. 15-27, 2008.

[51] D. Matignon, "Stability results for fractional differential equations with applications to control processing," in Proceedings of the Computational Engineering in Systems Applications, vol. 2, pp. 963-968, Lille, France, 1996. 


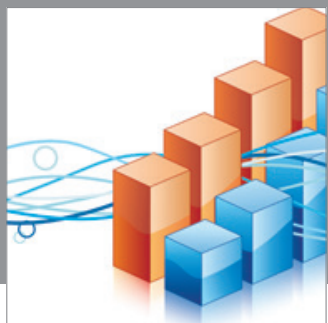

Advances in

Operations Research

mansans

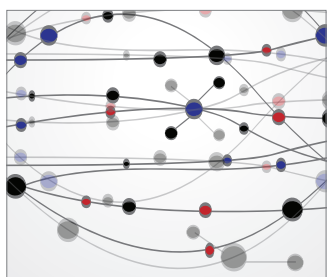

The Scientific World Journal
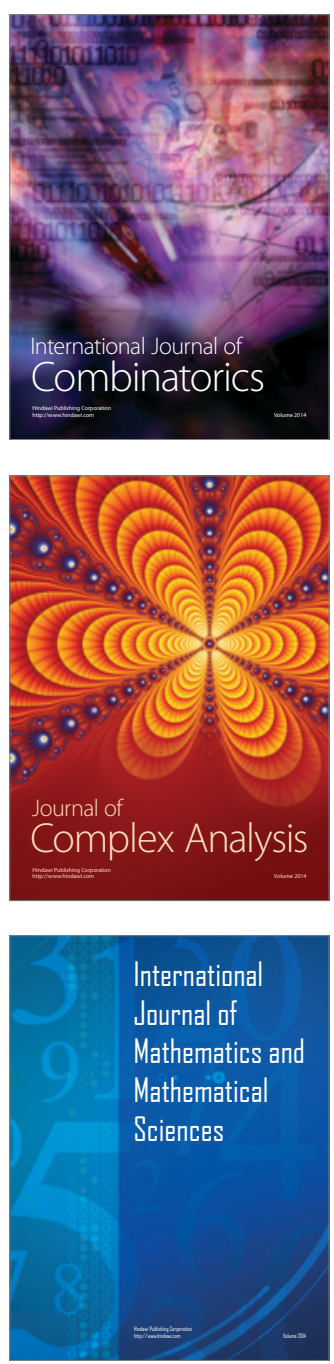
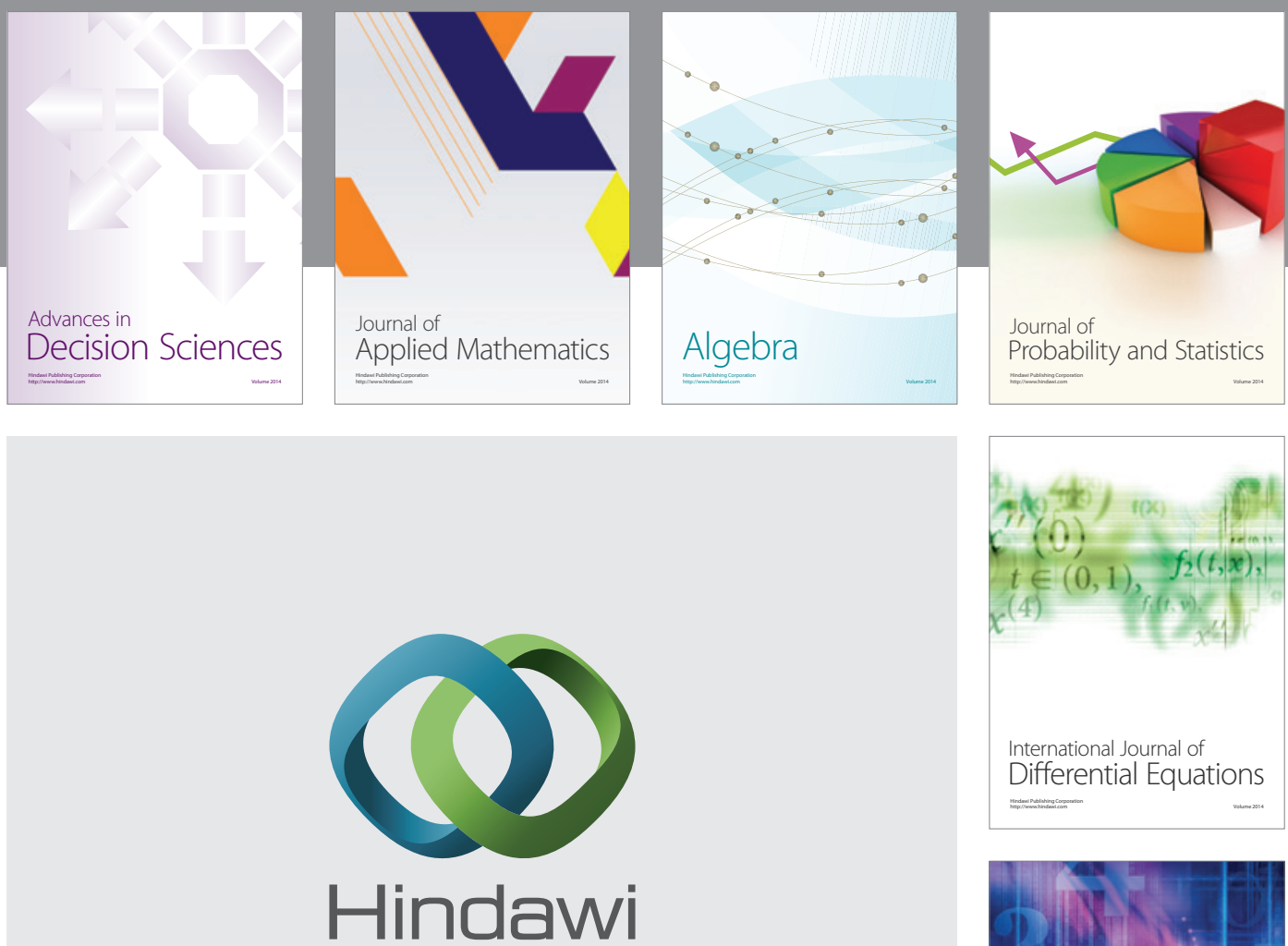

Submit your manuscripts at http://www.hindawi.com
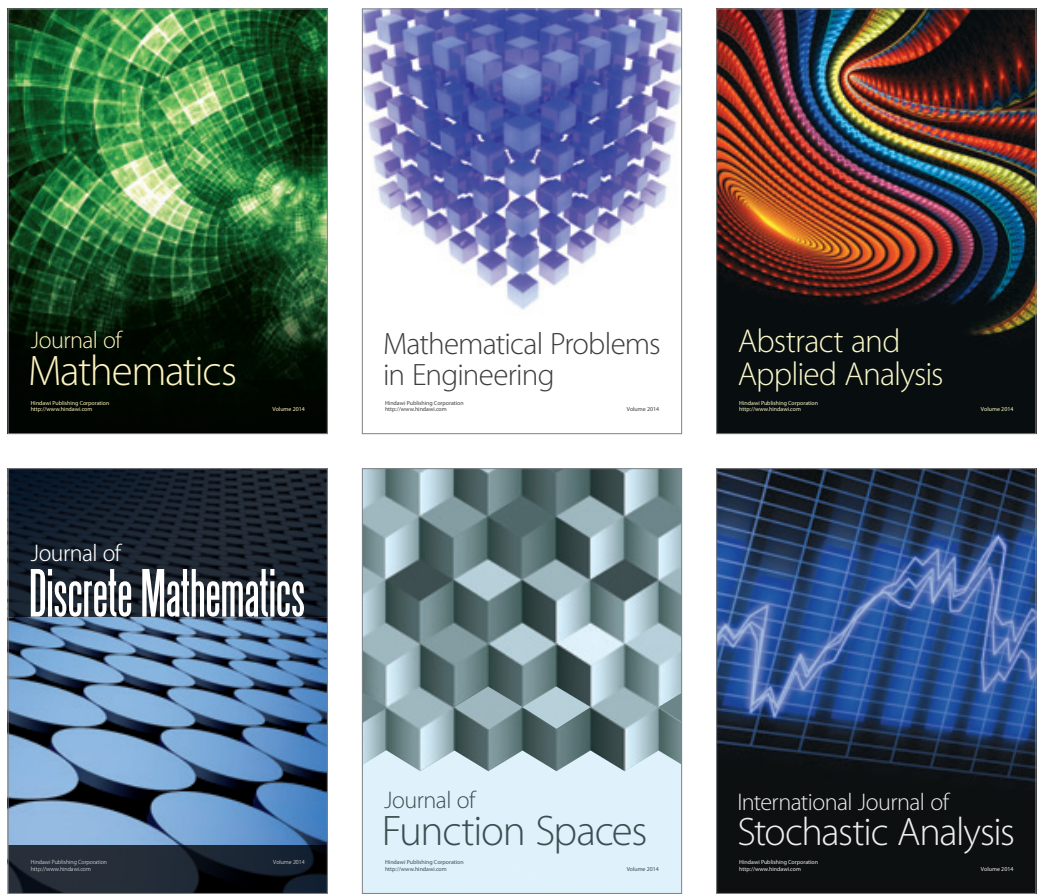

Journal of

Function Spaces

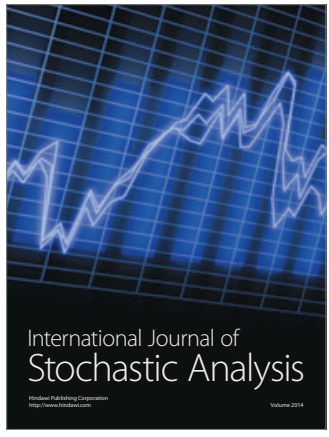

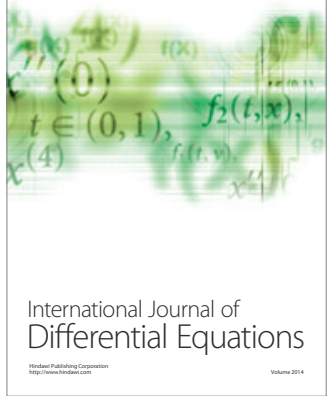
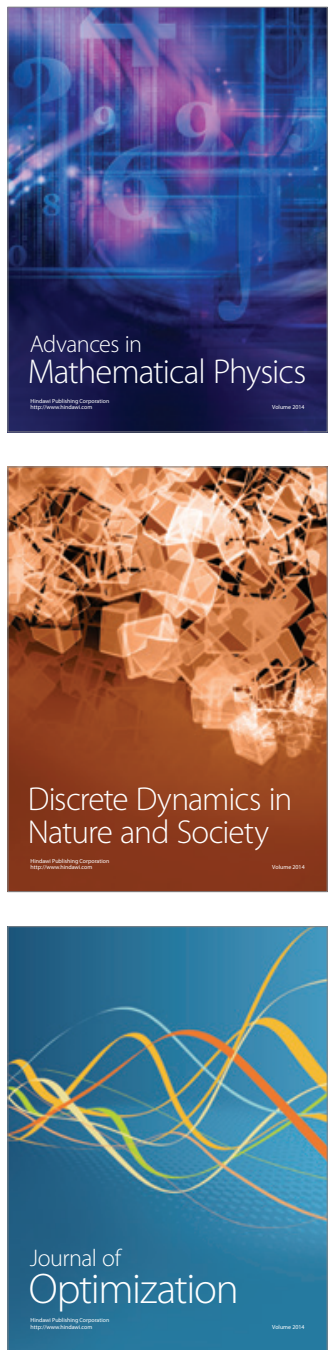